Journal of Computer Science 7 (7): 1011-1019, 2011

ISSN 1549-3636

(C) 2011 Science Publications

\title{
Performance Analysis of Congestion Reduction Routing in Wireless Sensor Networks
}

\author{
T.V.P. Sundararajan, Thiyaneswaran Manoharan and Abinaya Rajendran \\ Bannari Amman Institute of Technology, \\ Erode, TamilNadu, India
}

\begin{abstract}
Problem statement: Data generated in wireless sensor networks may not all be alike: some data may be more important than others and hence may have different delivery requirements, To solve this problem addressed a differentiated data delivery in the presence of congestion in wireless sensor networks and proposed a class of algorithms that enforce differentiated routing based on the congested areas of a network and data priority. Approach: The basic protocol, called Congestion-Reduction Routing (CRR), discovers the congested zone of the network that exists between high-priority data sources and the data sink and using simple forwarding rules, dedicates this portion of the network to forwarding primarily high-priority traffic. Since CRR requires some overhead for establishing the high-priority routing zone, it is unsuitable for highly mobile data sources. To accommodate all these things defined MAC-Enhanced CRR (MCRR), which includes MAC-layer enhancements and a protocol for forming high-priority paths on the fly for each burst of data. MCRR effectively handles the mobility of high-priority data sources, at the expense of degrading the performance of low-priority traffic and presented an extensive simulation results for CRR and MCRR and an implementation of MCRR on a 48-node testbed. Results: Proposed CRR and MCRR algorithms were implemented by using NS2 simulator and the QOS parameters on throughput, packet delivery ratio, delay and energy. All parameters were analyzed and compared with basic AODV mechanism. Conclusion/Recommendations: CRR is better suited for static networks with long-duration HP floods. For bursty HP traffic and/or mobile HP sources, MCRR is a better fit. Because of the lower delay, CRR and its variants appear suitable to real-time data delivery.
\end{abstract}

Key words: Wireless sensor networks, High-Priority (HP), Packet Delivery Ratio (PDR), HP data, QOS parameters, Packet Delivery Ratio (PDR), Low-Priority (LP), LP packets

\section{INTRODUCTION}

With large deployment sizes, congestion becomes an important problem. Congestion may lead to indiscriminate dropping of data (i.e., High-Priority (HP) packets may be dropped while Low-Priority (LP) packets are delivered. It also results in an increase in energy consumption to route packets that will be dropped downstream as links become saturated. As nodes along optimal routes are depleted of energy, only nonoptimal routes remain, further compounding the problem. To ensure that data with higher priority is received in the presence of congestion due to LP packets, differentiated service must be provided. In this work, we are interested in congestion that results from excessive competition for the wireless medium. Existing schemes detect congestion while considering all data to be equally important. We characterize congestion as the degradation of service to HP data due to competing LP traffic. In this case, congestion detection is reduced to identifying competition for medium access between HP and LP traffic. Congestion becomes worse when a particular area is generating data at a high rate. This may occur in deployments in which sensors in one area of interest are requested to gather and transmit data at a higher rate than In this case, routing dynamics can lead to congestion on specific paths. These paths are usually close to each other, which lead to an entire zone in the network facing congestion (Alfawaer et al., 2007; Hull et al., 2004; Sharieh et al., 2008). We refer to this zone, essentially an extended hotspot, as the congestion zone. In this project, we examine data delivery issues in the presence of congestion. We propose the use of data prioritization and a differentiated routing protocol and/or a prioritized medium access scheme to mitigate its effects on HP traffic. We strive for a solution that accommodates both LP and HP traffic when the network is static or near static and enables fast recovery of LP traffic in networks with mobile HP data sources.

Corresponding Author: T.V.P. Sundararajan, Bannari Amman Institute of Technology, Erode, TamilNadu, India 


\section{J. Computer Sci., 7 (7): 1011-1019, 2011}

Our solution uses a differentiated routing approach to effectively separate HP traffic from LP traffic in the sensor network. HP traffic has exclusive use of nodes along its shortest path to the sink, whereas LP traffic is routed over uncongested nodes in the network but may traverse longer paths. Our contributions in this work are listed as follows.

Design of CRR routing protocol: This protocol provides a network-layer solution to provide differentiated service in congested sensor networks. It also prevents severe degradation of service to LP data by utilizing uncongested parts of the network.

Design of MAC-Enhanced CRR (MCRR): MCRR is primarily a MAC-layer mechanism used in conjunction with routing to provide mobile and lightweight conzones to address sensor networks with mobile HP data sources and/or bursty HP traffic. Compared to CRR, MCRR has a smaller overhead but degrades the performance of LP data more aggressively.

We compare CRR and MCRR to an AODV scheme enhanced with priority queues (AODV\&PQ). Both CRR and MCRR lead to a significant increase in the successful packet delivery ratio of HP data and a clear decrease in the average delivery delay compared to AODV\&PQ. CRR and MCRR also provide low jitter. Moreover, they use energy more uniformly in the deployment and reduce the energy consumed in the nodes that lie on the conzone, which leads to an increase in connectivity lifetime. In the presence of sufficient congestion, CRR also allows an appreciable amount of LP data to be delivered. We further show that, in the presence of mobile HP data sources, MCRR provides mobile conzones, which follow the HP traffic.

Related work: An obvious solution to enhance service to $\mathrm{HP}$ data is to use priority queues to provide differentiated services. However, in such schemes, though HP packets get precedence over LP packets within a node, at the MAC layer, they still compete for a shared channel with LP traffic sent by surrounding nodes. As a result, without a routing scheme to address the impact of congestion and hotspots in the network, local solutions like priority queuing is not sufficient to provide adequate priority service to important data. QoS in sensor networks (Thenmozhi and Rajaram, 2011) has been the focus of current research. Existing work provides soft real-time guarantees for end-to-end traffic using feedback control and location awareness. It also concludes that local adaptation at the MAC layer (Singh et al., 2007) alone is insufficient to address the problem of hotspots and that routing is essential to the solution. An energy-aware QoS routing protocol (Thenmozhi and Rajaram, 2011) to support the delivery of real-time data in the presence of interfering non-realtime data by using multiple queues in each node in a cluster-based network; they do not consider the impact of congestion in the network and the interference that non-real-time traffic can cause to real-time data. Existing work on MAC Layer addresses the issue of increased traffic intensity in the proximity of a sink by using a schedule based and contention-based MAC hybrid. As with data aggregation schemes, it serves to delay the occurrence of congestion. Back pressure and rate limiting are essential to avoid situations where the network capacity is less than the amount of traffic being injected into the medium. But, Existing schemes do not adopt differentiated routing. Also, in a large network that is under congestion in a constrained area, our approach leverages the large uncongested parts of the network that is often underutilized to deliver LP traffic.

Existing works on congestion (Jasem et al., 2009) in sensor networks have two aspects: detection and mitigation. Existing systems use velocity monotonic scheduling. Applications assign an expected speed to each data packet, which is then ensured by these schemes. The speed that the application should assign to a packet if the network is congested is unclear. These schemes spread traffic around hotspots, but they do not give preference to HP data. In fact, if LP data has led to a hotspot in an area, routes for HP data that later enter the network will circumvent this hotspot. This will increase the number of hops over which this data has to be routed and increase the energy consumed in the network. In the worst case, no path for HP data may be found and these packets will be dropped. Additionally, Existing scheme achieves reliability by duplicating packets and routing them over different paths to the destination. Duplication of packets in congested networks may further precipitate congestion. Also, these schemes do not explicitly separate LP and HP traffic generated in the same area.

Though these schemes (Jasem et al., 2009) take important steps to mitigate congestion in sensor networks, they treat all data equally. These schemes are complementary to the capability provided by our protocol. Similarly, our solutions do not preclude the use of priority queues, which can be added as a simple extension.

\section{MATERIALS AND METHODS}

Congestion reduction routing: An example of the problem scenario that we consider is shown in Fig. 1. 


\section{J. Computer Sci., 7 (7): 1011-1019, 2011}

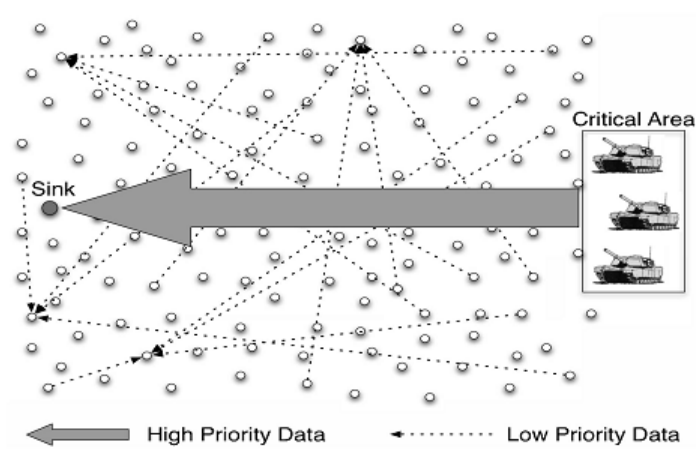

Fig. 1: A critical area of a sensor network may generate HP data at a high rate. This causes congestion in a part of the network exacerbated by the presence of LP data being routed in that area

An important event occurs in one portion of the sensor field, which we call the critical area. This critical area will typically consist of multiple nodes. In such a scenario, there is a data processing center for collecting sensitive information from the critical area. Such data is assigned a higher priority than other data. There might also be several nodes collecting different types of LP information from other parts of the network. In the presence of this background LP traffic, without differentiating between the two priority classes, congestion will degrade the service provided to HP data. This may result in HP data being dropped or delayed so long that it is of no use to the data processing center. We refer to the area that contains the shortest paths from the critical area to the sink as the congestion zone. HP data would ideally traverse the congestion zone but will face competition for medium access due to LP traffic.

Our basic solution, called Congestion Reduction Routing (CRR), operates solely in the network layer. Packets are classified as HP or LP by the data sources and nodes within a congestion zone only forward HP traffic. LP traffic is routed out of and/or around the congestion zone. In effect, we segment the network into two parts by using forwarding rules. One limitation with this system is that it requires some overhead to discover the congestion zone. While this overhead is reasonable, it may still be too heavy weight if the data source is moving often and the congestion zone is changing frequently or if the HP traffic is short lived. Hence, CRR is designed for static or nearly static networks with long-lived HP flows.

CRR uses the enhanced AODV with Priority queuing technique to study the performance of routing mechanism. Since it involves the data prioritization on both high and low priority, it requires priority queuing on AODV.

CRR comprises three steps: HP network formation, congestion zone discovery and differentiated routing. The combination of these functions segments the network into on-congestion zone and off-congestion zone nodes. Only HP traffic is routed by on-congestion zone nodes. Note that the protocol specifically accommodates LP traffic, albeit with less efficient routes than HP traffic.

For the purposes of this discussion, we assume that there is one HP sink and a contiguous part of the network (critical area) that generates HP data in the presence of network wide background LP traffic. We also assume that nodes are location aware and densely deployed with uniform distribution.

Since nodes in the scenario in Fig. 1 send all HP data to a single sink, tree-based routing, with the HP sink being the root, is most appropriate. However, treebased routing schemes suffer from congestion, especially if the number of messages generated at the leaves is high. This problem becomes even worse when we have a mixture of LP and HP traffic traveling through the network. Therefore, even when the rate of HP data is relatively low, the background noise created by LP traffic will create a congestion zone that spans the network from the critical area to the HP sink. Due to this congestion, service provided to HP data may degrade and nodes within this area may die sooner than others, leading to only suboptimal paths being available for HP data, or a network partition may result, isolating the sink from the critical area.

MAC-enhanced congestion reduction routing: Here we presented MCRR, a combined MAC and routing scheme designed to support situations in which critical events may move or the sensors generating HP data may move. Though conzone discovery is dynamic in CRR, the overhead required to maintain the HiNet in a dynamic environment may be prohibitive. As a result, we use a lightweight dynamic differentiated routing mechanism to accommodate mobile data sources. MCRR is based on MAC-layer enhancements that enable the formation of a conzone on the fly with each burst of data. The trade-off is that it effectively preempts the flow of LP data, thereby seriously degrading its service.

Unlike CRR, MCRR does not form an HP network. Instead, HP paths are dynamically created, since the sources (or the sinks) are expected to be mobile. Thus, MCRR discovers the conzone while discovering the paths from HP sources to the sink. 


\section{J. Computer Sci., 7 (7): 1011-1019, 2011}

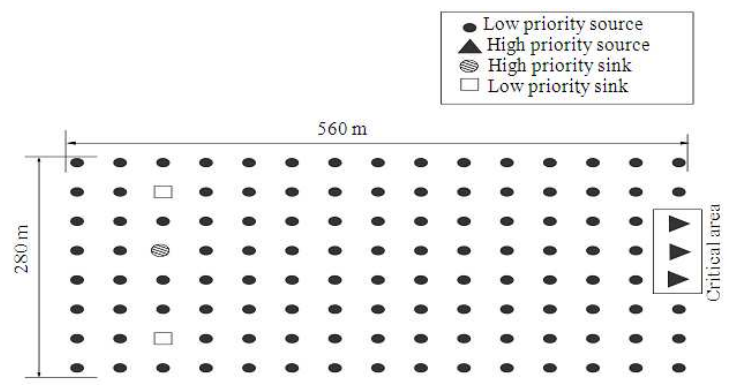

Fig. 2: Simulation scenario

The enhanced MAC-layer of MCRR uses an RTS/CTS protocol that is augmented to carry information about the priority level of the data being transferred. Each RTS and CTS packet is tagged with a priority level. During channel contention, if a node has HP data to send and overhears an LP RTS, it jams the channel with an HP CTS, causing nodes forwarding LP data to back off. Furthermore, if a node with LP data overhears an HP RTS or CTS, it will back off the channel, as described here.

Though $802.11 \mathrm{e}$ is similar to MCRR in that they both prioritize access to the medium, the prioritized RTS/CTS messages in highly congested networks may be dropped. 802.11e's policy of guarding every transmission with an RTS/CTS exchange leads to a prohibitive overhead. Woo and Culler state that RTS/CTS exchange imposes an overhead of up to 40 percent. The extent of overhead experienced depends on the relative size of the RTS/CTS packets and the data packets. In sensor networks, data packet sizes are not large enough to justify the cost of RTS/CTS exchange to guard every packet. Hence, 802.11 e is unsuitable for sensor networks. MCRR uses a silencing mechanism that does not require preempting all LP data transmissions in the neighborhood for each HP data to be sent. Rather, MCRR silences the conzone and its neighborhood during route discovery and/or maintenance.

Though the cost of an RTS/CTS exchange for each data packet may be considerable for a sensor network, even S-MAC, a widely used MAC scheme for sensor networks, uses one RTS/CTS exchange for a collection of message fragments. Similarly, the cost of RTS/CTS imposed by MCRR is not prohibitive, since it uses these RTS/CTS packets only during the route discovery/maintenance phase. Hence, the scalability of the RTS/CTS overhead for MCRR is not an issue.

In MCRR, nodes discover if they are on the conzone by using the conzone discovery explained in the following. Like CRR, this conzone discovery is triggered when an area starts generating HP data. For the conzone to be discovered dynamically, MCRR uses two timers to regulate when a node decides it is no longer part of the HP path. One timer, called the overhearing timer, monitors how long it has been since the last HP packet was heard. This timer is used to control nodes in the communication range of the conzone but that are not necessarily involved in forwarding the packets. The overhearing timer is reset any time an HP packet is overheard or any time an HP packet is received (since nodes involved in forwarding packets are clearly within the communication range of nodes transmitting those packets). The second timer, called the received timer, controls nodes either generating or forwarding HP data.

In MCRR, each node in the network can be in one of three states, dictating whether it is a part of the conzone or not or within the communication range of the conzone but not a part of it. This last mode creates a shadow area that separates HP traffic from LP traffic.

\section{RESULTS}

Simulation setup: The simulations were conducted in Network simulator NS2 with version 2.31, with a deployment area of $560 \mathrm{~m}$ by $280 \mathrm{~m}$. In this area, 120 nodes are placed as shown in Fig. 2, with the separation between neighboring nodes along both axes being 40 $\mathrm{m}$. Note that we use grids as deployments in this project to emulate uniformly dense deployments and such grids are not a requirement of our algorithms. As long as the neighborhood relationships are similar, the results will not differ significantly from those presented in this project.

Two LP sinks receive all LP data, while a single sink receives all HP data. Three nodes form the critical area and send HP data. The rest of the nodes, other than the three sinks and the three critical area nodes, send LP data to either LP sink. This LP data serves as the background traffic in our simulations. Note that the HP sources in our simulations were placed at the edge of the deployment to get a sufficient number of hops from them to the HP sink. In a large deployment of hundreds of nodes, these HP sources need not be at the edge of the deployment. Results were recorded when the system reached a steady state. CRR uses AODV to route LP data outside the congestion zone, with a modification to ensure that off congestion zone nodes do not route such data into the congestion zone.

We compare CRR to an enhanced version of AODV that we implemented, that is, AODV\&PQ. AODV\&PQ maintains two queues at each node. The first is an HP queue. Messages in this queue are transmitted if present. 
The second queue is an LP queue. When the HP queue is empty, messages from this queue are transmitted. This policy provides absolute privilege to HP data within a node. AODV\&PQ is a simple generalization of priorityqueue based schemes.

In our environment of large multihop networks, DSR fails to route any HP data successfully. DSR is intended to work over networks with a small number of hops. Similarly, Directed Diffusion was unable to route any HP data successfully due to the large control overhead involved in the initial flooding that is required to set up the data paths. One-Phase Pull Filter was used in the simulations and though it is expected to route LP packets successfully, our simulations showed that as the number of senders in the deployment was increased beyond 10, Directed Diffusion failed to route any data. As with DSR, Directed Diffusion is not intended for such applications. It was mainly designed to work in cases where the number of sinks and senders is small.

Performance metrics: Average no of received packets: This parameter explains about the no of packets received at particular simulation time. The results are being taken at various simulation times from $0-100$ seconds.

Packet Delivery Ratio (PDR): PDR is the most important metric that we should consider in packet forwarding. It may affects by different criteria such as packet size, group size, action range and mobility of nodes.PDR gives about to the successful delivery of packets to destination from acknowledgements received.

Average delay: Average end to end delay includes all possible delays caused by buffering during route discovery latency, queuing at the interface queue, retransmission delays at the MAC and propagation and transfer times of data packets.

Energy: This is one of the most essential QOS parameter in wireless networks on nodes energy consumption. This energy consumption is taken on transmit, receive and idle modes.

\section{DISCUSSION}

The CRR and MCRR Routing Simulation is being taken for the QOS parameters of no of received packets, Packet Delivery Ratio (PDR) on both HP and LP datas, Energy, delay, Routing overhead and routing load.

In data transmission, throughput is the amount of data moved successfully from one place to another in a given time period. Throughput or network throughput is the average rate of successful message delivery over a communication channel.

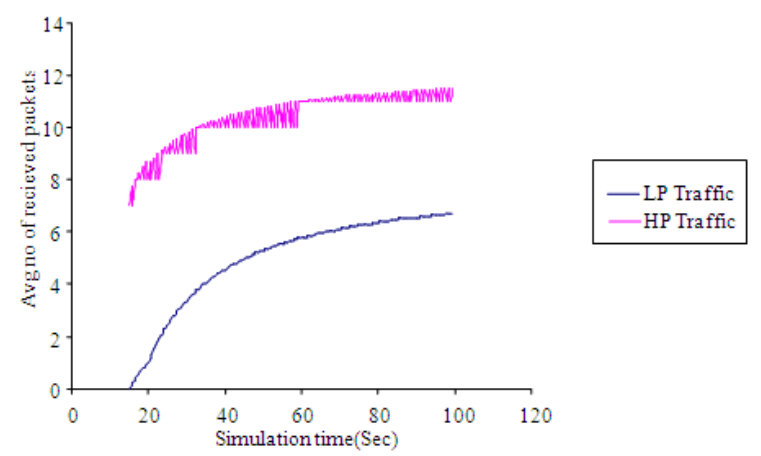

Fig. 3: Average no of received packets for CRR

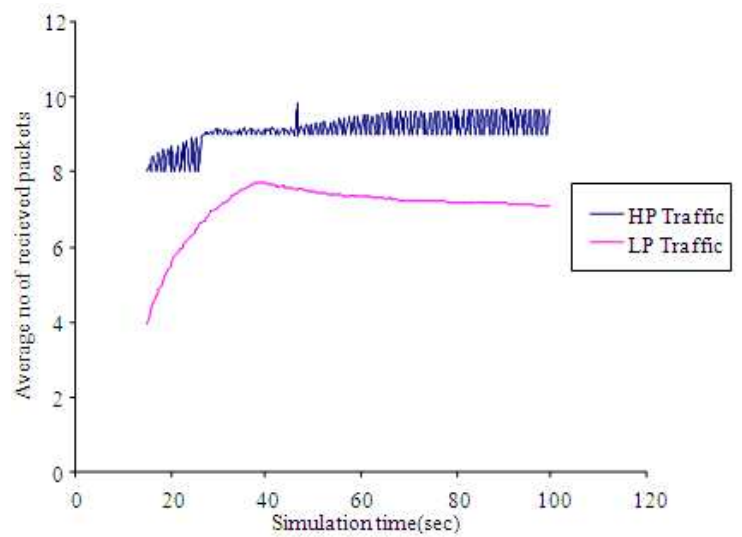

Fig. 4: Average no of received packets for MCRR

Received packets plot is being plotted between Simulation time (Vs.) Avg no of received packets at receiver. PDR is being plotted against various Simulation time intervals from 0-100 Sec.

From Fig. 3-4 it is observed that In LP Traffic the average no of received packets are increasing against the simulation time likewise AODV throughput also is increasing against the offered load (Kbits/Sec) depending upon the no of nodes with no of source nodes. This LP traffic is not too much affected by congestion so that it is gradually increasing against the time.

In HP traffic becomes with an oscillations because the HP traffic is being happened in the congested Zone area so that it cannot be able to provide the consistent performance on this QOS parameter. It provides the oscillations response on average no of packets against the simulation time.

From Fig. 5 it is shown that PDR of LP Traffic becomes with a tiny oscillations against time between 20-30 sec of time because packets are transmitted in the out of congestion area. After $30 \mathrm{sec}$ of time the 


\section{J. Computer Sci., 7 (7): 1011-1019, 2011}

performance of PDR becomes fairly consistent so that it shows as the consistent delivery of packets to the sink. In between 10-20 sec the performance is showing rapid growth due to LP traffic congestion in off congested zone area. This shows that CRR is protecting from the severe degradation of LP traffic.

PDR of HP Traffic becomes with tiny variations in PDR at time period of 15-30 sec after that it becomes mostly consistent throughout the time period Likewise AODV performance has decreasing PDR against speed $(\mathrm{m} / \mathrm{s})$ depending upon the number of nodes with no of source nodes.

When compared the AODV performance with CRR, CRR provides the significant amount of increasing PDR whereas AODV has increasing PDR against the time. Due to Consistent delivery of PDR in HP data shows that the congestion is being minimized in the CRR algorithm.

From Fig. 6 Consistent delivery of PDR in HP Traffic shows that the congestion is being minimized in the MCRR algorithm. MCRR has an advantage of degrading LP Traffic mostly which is being shown from PDR plot where it has very low PDR of data throughout the simulation period.

From the Fig. 7 it is noted that CRR has taken less average delay against the simulation time compared to AODV.So that CRR provides faster routing of packets to the destination. At simulation time $60 \mathrm{sec}$ AODV took additional $33.9 \%$ of average delay compared to CRR.MCRR has taken lesser delay when compared to MCRR.

From Fig. 8 it is observed that According to simulation the HP data delivery took 1.45 milliseconds of time in order to complete its transmission. Likewise the LP data delivery took 3.92 milliseconds of time in order to complete its transmission whereas AODV took 8.28 milliseconds of time in order to complete its transmission. So that it concludes that CRR is somewhat suffering from LP traffic degradation.

From Fig. 9 When Comparing the CRR's delivery delay of LP and HP Traffic with MCRR, MCRR took lesser delivery delay. So that it increases the operating lifetime of nodes.

From Fig. 10 End to End delay becomes very smaller in MCRR compared with CRR. Almost $45 \%$ reduction of delay for MCRR compared with CRR so that it increases the operating lifetime of nodes.

The Energy QOS parameter is being considered for the entire network also this QOS is being calculated based upon the energy consumption by each node. So that it does not consider about the prioritization of network in energy QOS parameter.
From Fig. 11 it is shown that The Average energy consumed for CRR routing is lesser than that of AODV routing. This QOS parameter is taken from the energies consumed by each node.

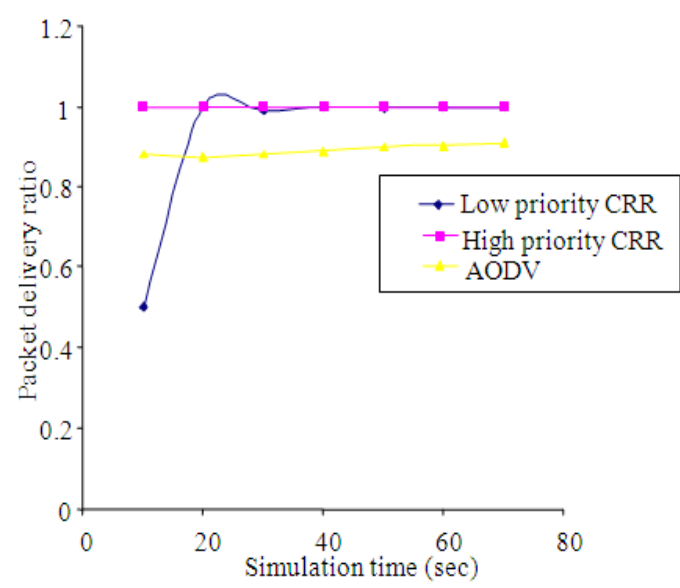

Fig. 5: PDR comparison CRR with AODV

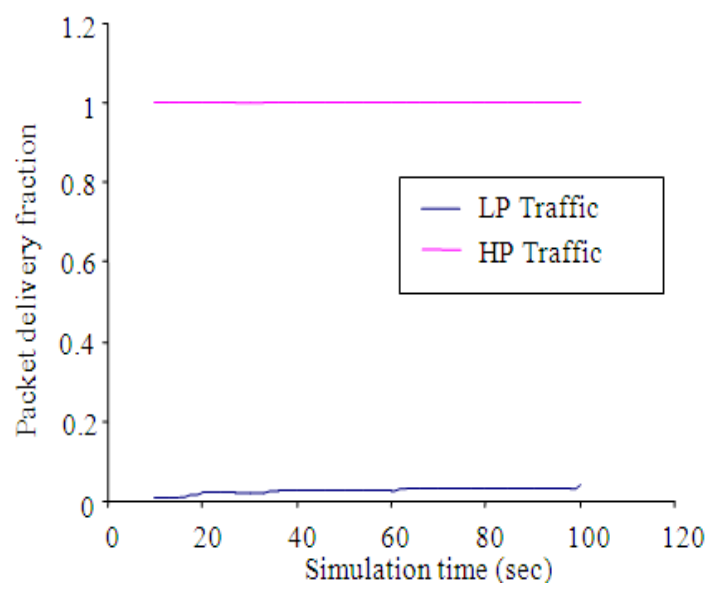

Fig. 6: PDR comparison of LP and HP traffic's CRR

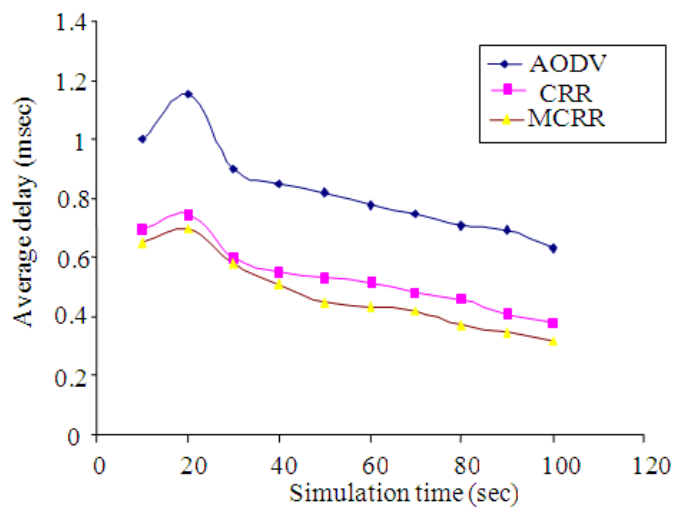

Fig. 7: Average delay 


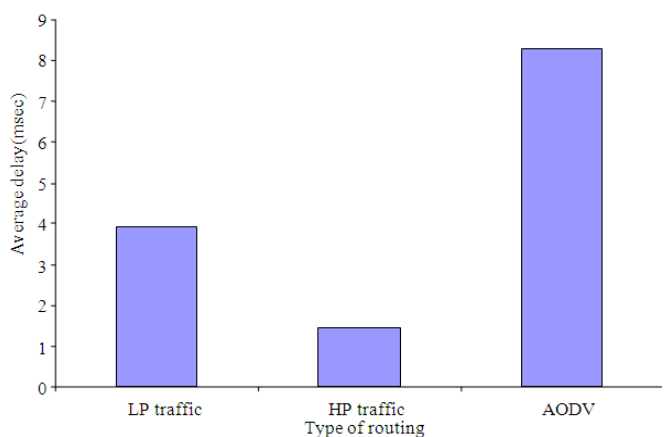

Fig. 8: Average delivery delay of CRR

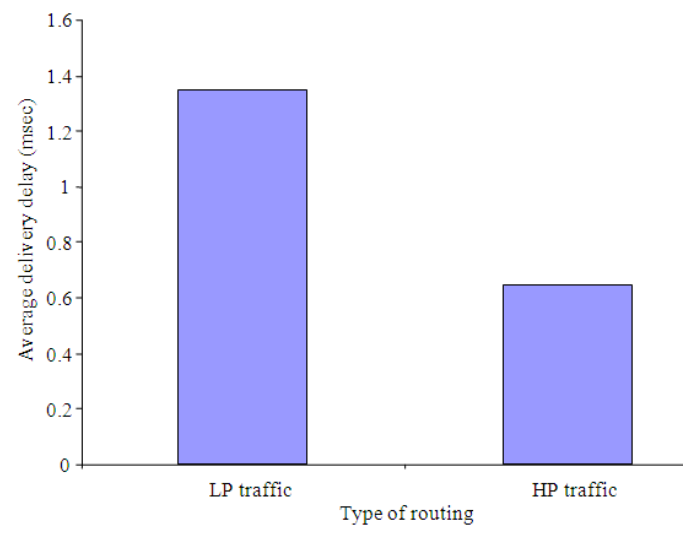

Fig. 9: Average delivery delay of MCRR

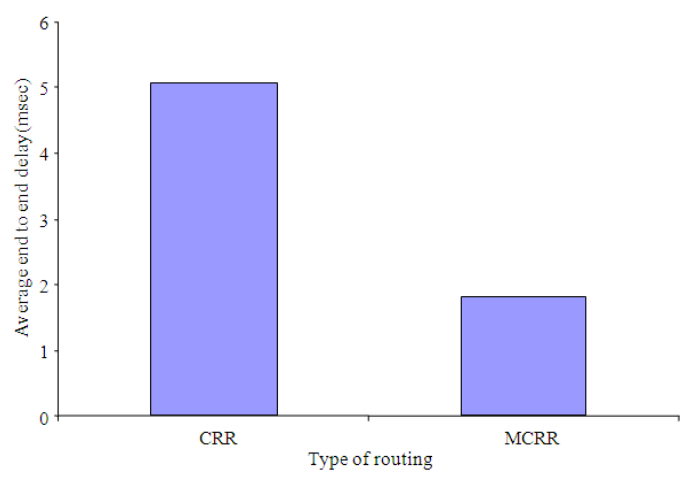

Fig. 10: Average end to end delay

CRR took around $9 \%$ of lesser average total energy consumption against AODV. When CRR's energy is compared with MCRR, MCRR took $8.5 \%$ lesser than that of CRR.

From Fig. 12 it is observed that MCRR took lesser energy consumption in transmit mode when compared with CRR and AODV.

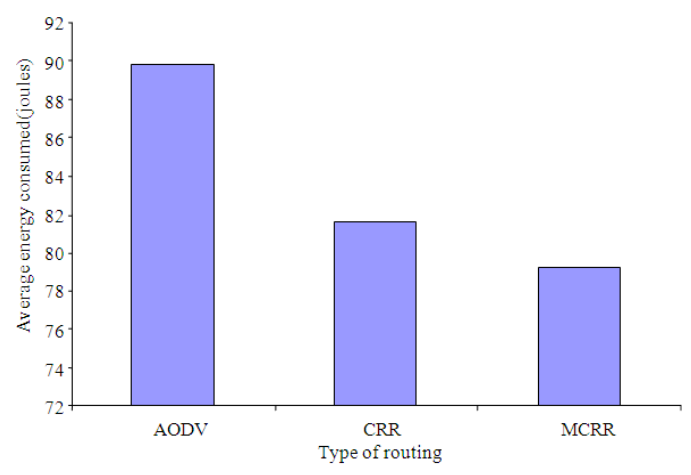

Fig. 11: Average Energy consumed

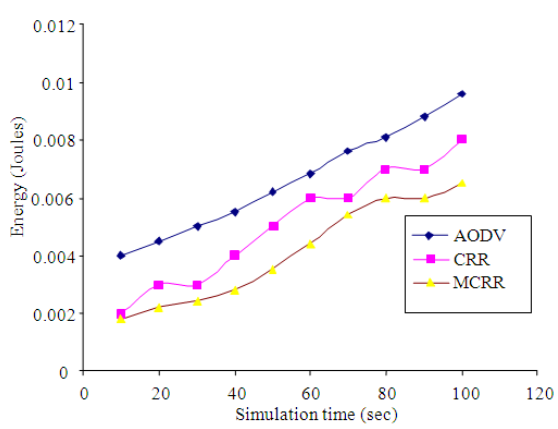

Fig. 12: Energy consumption in transmit mode

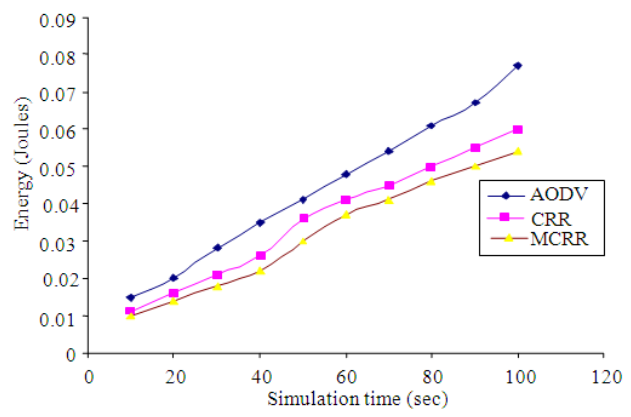

Fig. 13: Energy consumption in receive mode

From Fig. 13 it is shown that The CRR Routing took lesser amount of energy consumption in receive mode compared to AODV.CRR took $22 \%$ of lesser amount of energy in receive mode compared to AODV at simulation time $100 \mathrm{sec}$. When simulation time increases, the energy consumption in Receive mode also increases in CRR and AODV. Likewise Energy in Receive mode MCRR took smaller consumption compared with CRR. For example at 60 seconds simulation time MCRR had taken $25 \%$ of lesser energy consumption compared with CRR. 


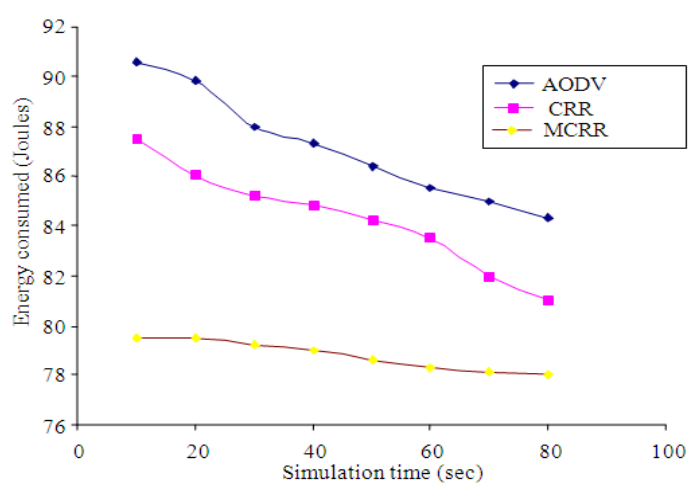

Fig. 14: Energy consumed (Vs) simulation time

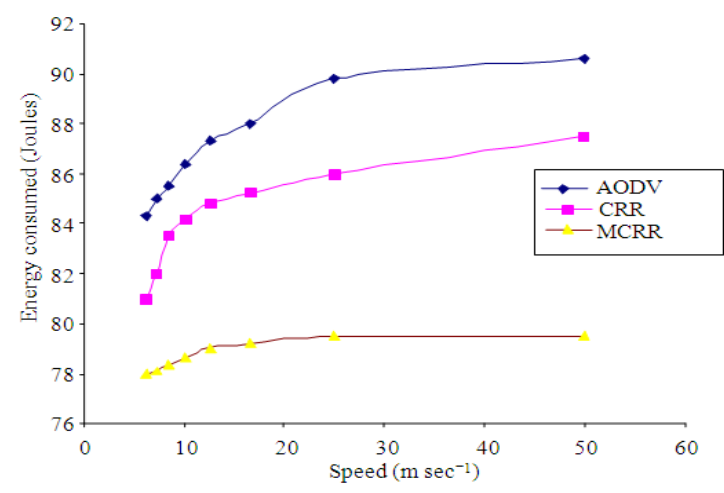

Fig. 15: Speed (Vs) energy consumed

From Fig. 14 it is observed that The energy consumed by both AODV and CRR has decreasing energy consumption against the time where as AODV took more energy consumption compared to CRR.CRR took less energy consumption so that it provides the increase in life time of nodes also mitigates the congestion. Likewise in Energy consumption plot MCRR took smaller consumption compared with CRR. For example at $60 \mathrm{sec}$ simulation time MCRR had taken $7 \%$ of lesser energy consumption compared with CRR. Normally Due to congestion in CRR took optimal shortest path tree based routing to reach sink so that it takes less energy consumption.

From Fig 15 it is noted that CRR took lesser energy consumption compared to AODV.AODV took $3.5 \%$ of more energy consumption compared with CRR against speed at $50 \mathrm{~m} \mathrm{sec}^{-1}$. When speed increases, the energy consumption also increases in CRR and AODV. Likewise in speed vs. energy plot MCRR took smaller consumption compared with CRR. For example at 40 $\mathrm{m} / \mathrm{s}$ speed MCRR had taken $12 \%$ of lesser energy consumption compared with CRR.

\section{CONCLUSION}

In this study, we addressed data delivery issues in the presence of congestion in wireless sensor networks. We proposed CRR, which is a differentiated routing protocol and uses data prioritization. We also develop MCRR, which deals with mobility and dynamics in the sources of HP data. Our extensive simulations show that as compared to AODV, CRR and its variants increase the fraction of HP data delivery and decrease delay for such delivery while using energy more uniformly in the deployment. CRR also routes an appreciable amount of LP data in the presence of congestion. We additionally show that MCRR maintains HP data delivery rates in the presence of mobility. This algorithm can be applied at weather monitoring system application as well as on body area networks. This routing algorithm took lesser energy consumption so that it increases the lifetime of nodes. Also it took lesser amount of average delay compared to AODV. Therefore CRR is better suited for static networks with long-duration HP floods. Both CRR and MCRR support effective HP data delivery in the presence of congestion. CRR is better suited for static networks with long-duration HP floods. For bursty HP traffic and/or mobile HP sources, MCRR is a better fit. Because of the lower delay, CRR and its variants appear suitable to real-time data delivery. To ensure QoS for video streams, reactive dropping methods could be combined into the routing protocol.

\section{REFERENCES}

Alfawaer, Z.M., G.W. Hua and N.A. Hamdeh, 2007. Utilization of AODV in wireless ad hoc networks. $\begin{array}{lcccc}\text { J. Comput. Sci., 6: } & \text { 218-222. } & \text { DOI: } \\ \text { 10.3844/jcssp.2010.1164.1169 } & \end{array}$

Hull, B., K. Jamieson and H. Balakrishnan, 2004. Mitigating congestion in wireless sensor networks. Proceedings of the Second ACM Conference Embedded Networked Sensor Systems, Nov. 0305, Baltimore, MD, USA., pp: 495-512. DOI: 10.1145/1031495.1031512

Jasem, H.N., Z.A. Zukarnain, M. Othman and S. Subramaniam, 2009. Experimental evaluation of bottleneck link utilization with new-additive increase multiplicative decrease congestion avoidance and control algorithm. J. Comput. Sci., pp: $\quad 1055-1059$. DOI: 10.3844/jcssp.2009.1055.1059 
Sharieh, A., M. Itriq and W. Dbabat, 2008. Dynamic resource synchronizer mutual exclusion algorithm for wired/wireless distributed systems. Am. J. Applied Sci., 5: 829-834. DOI: 10.3844/ajassp.2008.829.834

Singh, G., A.P.S. Atwa and B.S. Sohis, 2007. Mobility management technique for real time traffic in 802.11 networks. J. Comput. Sci., 3: 390-398.

DOI: $10.3844 /$ jcssp.2007.390.398
Thenmozhi, D.S. and M. Rajaram, 2011, An effective contention aware stable path finding approach to provide quality of service in mobile adhoc networks. J. Comput. Sci.. 7: 352-358. DOI: 10.3844/jcssp.2011.352.358 